Published in final edited form as:

J Proteome Res. 2016 April 1; 15(4): 1205-1212. doi:10.1021/acs.jproteome.5b01089.

\title{
A Redox Imbalance Underlies the Fitness Defect Associated with Inactivation of the Pta-AckA Pathway in Staphylococcus aureus
}

\author{
Darrell D. Marshall ${ }^{1, \dagger}$, Marat R. Sadykov ${ }^{2, \dagger}$, Vinai C. Thomas ${ }^{2}$, Kenneth W. Bayles ${ }^{2}$, and \\ Robert Powers ${ }^{1,}{ }^{*}$ \\ ${ }^{1}$ Department of Chemistry, University of Nebraska-Lincoln, Lincoln, NE 68588-0304 \\ 2Department of Pathology and Microbiology, University of Nebraska Medical Center, Omaha, NE \\ $68198-5900$
}

\begin{abstract}
The phosphotransacetylase-acetate kinase (Pta-AckA) pathway is thought to be a vital ATP generating pathway for Staphylococcus aureus. Disruption of the Pta-AckA pathway during overflow metabolism causes significant reduction in growth rate and viability, albeit not due to intracellular ATP depletion. Here we demonstrate that toxicity associated with inactivation of the Pta-AckA pathway resulted from an altered intracellular redox environment. Growth of the pta and ack $A$ mutants under anaerobic conditions partially restored cell viability. NMR metabolomics analyses and ${ }^{13} \mathrm{C}_{6}$-glucose metabolism tracing experiments revealed the activity of multiple pathways that promote redox (NADH/NAD ${ }^{+}$) turnover to be enhanced in the pta and ackA mutants during anaerobic growth. Restoration of redox homeostasis in the pta mutant by overexpressing Llactate dehydrogenase, partially restored its viability under aerobic conditions. Together our findings suggest that during overflow metabolism the Pta-AckA pathway plays a critical role in preventing cell viability defects by promoting intracellular redox homeostasis.
\end{abstract}

\section{Graphical abstract}

\footnotetext{
*To whom correspondence should be addressed. Robert Powers, University of Nebraska-Lincoln, Department of Chemistry, 722 Hamilton Hall, Lincoln, NE 68588-0304, ; Email: rpowers3@unl.edu, Phone: (402) 472-3039, Fax: (402) 472-9402.

†qual contribution

Supporting information

The Supporting Information is available free of charge on the ACS Publications website. Supplementary figures demonstrating that the inactivation of the Pta-AckA pathway impairs growth of $S$. aureus.

Author Contributions

D.D.M., M.R.S., V.C.T., R.P. and K.W.B. designed the experiments, analyzed the data and wrote the manuscript; D.D.M., M.R.S. and V.C.T. performed the experiments.

Notes

The authors declare no competing financial interest.
} 

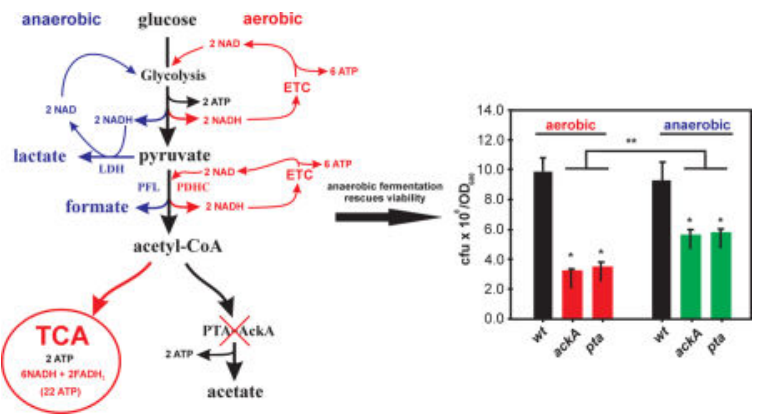

\section{Keywords}

Pta-AckA pathway; metabolomics; redox imbalance; NMR; Staphylococcus aureus

\section{INTRODUCTION}

Staphylococcus aureus is a versatile human pathogen responsible for a variety of infections that range from folliculitis to life-threatening diseases such as severe sepsis, endocarditis and bacteremia. ${ }^{1-3} S$. aureus infections represent an enormous challenge to physicians because of the emergence and dissemination of multidrug-resistant strains in the health care setting. ${ }^{1,2}$ The ability of this bacterial pathogen to survive and efficiently colonize diverse host environments is based on its proficiency to optimize virulence factor production and adjust its metabolism to rapid environmental changes. ${ }^{4-9}$

When grown under aerobic conditions, $S$. aureus primarily metabolizes glucose to acetate through the Pta-AckA pathway. In a recent study, we observed that inactivation of the PtaAckA pathway, resulted in poor growth and reduced viability at the exponential phase. Although carbon flux through the Pta-AckA pathway is used to generate ATP, the aerobic growth and viability defects associated with the pta and ackA mutants could not have resulted from ATP depletion as these strains surprisingly exhibited increased levels of intracellular ATP, presumably through increased glycolytic flux and redirection of carbon into the TCA cycle. ${ }^{10}$ One possibility for the altered growth and viability defects associated with the pta and ack $A$ mutants may arise from an altered intracellular redox environment resulting from inflated concentrations of $\mathrm{NAD}^{+}$and NADH. ${ }^{10}$

In the present study, we address this hypothesis by comparing the growth characteristics and metabolic changes in the pta and ack $A$ mutants relative to their isogenic wild-type strain following cultivation under aerobic and anaerobic conditions. We demonstrate that although growth defects due to pta and ack $A$ mutations persisted under both aerobic and anaerobic growth, cell viability relative to the wild type strain could be partially restored under fermentative growth. Metabolic differences associated with fermentative growth of the pta and ack $A$ mutants were determined by NMR spectroscopy and the observed metabolic changes support a role for the normalization of cellular redox status in the restoration of cell viability in the pta and ack $A$ mutants. Finally, confirming a strong relationship between cellular redox status and cell viability, the increased cell death in the pta mutant under 
aerobic growth could be partially restored to the wild-type levels by increasing NADH turnover following overexpression of the $1 d h 1$ gene in this mutant.

\section{MATERIALS AND METHODS}

\section{Bacterial strains, plasmids and growth conditions}

The construction of the ack $A$ and pta mutants in $S$. aureus strain UAMS-1 was described previously. ${ }^{10}$ The plasmid, pMRS110, containing the $l d h 1$ gene under the control of the cadmium-inducible promoter $\mathrm{P}_{c a d C}$ was constructed by amplifying 1-kb region from the $S$. aureus UAMS-1 chromosome using the primers, PstI-RBS-ldh1-f (GTCTCTGCAGCATAAGGAGGAATTTGTAATGAACAAATTTAAAGGGAACAAAGT) and ldh1-r (GGGGTAAGGTTTTACAATTTTTGGAATGG), and inserting the resulting DNA fragment into the PstI and SmaI sites of the shuttle vector, pBK123. ${ }^{11}$

$S$. aureus strains were grown in tryptic soy broth (TSB) without dextrose (BD Biosciences) supplemented with $0.25 \%$ glucose (Sigma-Aldrich) or on TSB containing agar. $S$. aureus cultures for both aerobic and anaerobic conditions were inoculated to 0.06 optical density at $600 \mathrm{~nm}\left(\mathrm{OD}_{600}\right)$ units from overnight cultures (grown in tryptic soy broth (TSB) without dextrose (BD Biosciences)), incubated at $37^{\circ} \mathrm{C}$, and aerated at $250 \mathrm{rpm}$ with a flask-tomedium ratio of 10:1. Bacterial growth was assessed by measuring the optical density at 600 $\mathrm{nm}$ or by determining the number of colony forming units (cfu) $\mathrm{ml}^{-1}$. Chloramphenicol was purchased from Fisher Scientific and was used at a final concentration of $10 \mu \mathrm{g} / \mathrm{ml}$.

\section{Measurement of extracellular glucose, acetic acid, and D, L- lactate}

Aliquots of bacterial cultures $(1 \mathrm{ml})$ were centrifuged for $3 \mathrm{~min}$ at $14,000 \mathrm{rpm}$ at $4^{\circ} \mathrm{C}$. The supernatants were removed and stored at $-20^{\circ} \mathrm{C}$ until use. Acetate, glucose, and D- and Llactate concentrations were determined using kits purchased from R-Biopharm according to the manufacturer's protocol.

\section{Determination of intracellular ATP concentrations}

Intracellular ATP concentrations were determined using the BacTiter-Glo kit (Promega). The kit was used according to the manufacturer's directions and as previously described. ${ }^{10}$ Metabolite concentrations were normalized to the number of viable-cell counts.

\section{NMR metabolomics sample preparation}

Samples for one-dimensional (1D) ${ }^{1} \mathrm{H}$ NMR and two-dimensional (2D) ${ }^{1} \mathrm{H}^{1}{ }^{13} \mathrm{C}$ heteronuclear single quantum coherence (HSQC) experiments were prepared from six and three replicate 50-mL cultures, respectively. $S$. aureus wild type strain UAMS-1, and mutant strains UAMS-1-ackA, and UAMS-1-pta were grown aerobically or anaerobically in TSB containing $0.25 \%$ glucose for $1 \mathrm{D}{ }^{1} \mathrm{H}$ NMR experiments or $0.25 \%\left[{ }^{13} \mathrm{C}_{6}\right]$ glucose (SigmaAldrich) for $2 \mathrm{D}^{1} \mathrm{H}_{-}{ }^{13} \mathrm{C}$ HSQC experiments. Bacterial cells were harvested during the exponential growth phase $(3 \mathrm{~h})$ after reaching an optical density of $10 \mathrm{OD}_{600}$ units. Samples were kept on ice through the entire preparation protocol. Bacterial cultures were centrifuged at $4^{\circ} \mathrm{C}$ for $7 \mathrm{~min}$ at $4,100 \mathrm{rpm}$ and then washed with ice cold $50 \mathrm{mM}$ phosphate buffer at $\mathrm{pH}$ 7.1. Enzymatic activity were quenched by suspending the cells in $700 \mu \mathrm{L}$ of ice cold ethanol 
[60\% ethanol, $40 \% \mathrm{D}_{2} \mathrm{O}$ (Isotec)]. ${ }^{12}$ The cells were then lysed using lysing matrix B tubes in a FastPrep instrument (Qbiogene). The lysates were centrifuged at $4^{\circ} \mathrm{C}$ for $5 \mathrm{~min}$ at 14,000 $\mathrm{rpm}$ to remove the cell debris. The samples were then lyophilized and suspended in $600 \mu \mathrm{L}$ of $99.8 \% \mathrm{D}_{2} \mathrm{O}$ phosphate buffer at $\mathrm{pH} 7.1$ (uncorrected) containing $50 \mathrm{mM}$ of (trimethylsilyl) propionic-2, 2, 3, 3- $\mathrm{D}_{4}$ acid sodium salt $\left(\mathrm{TMSP}-\mathrm{D}_{4}\right.$ ) for $1 \mathrm{D}^{1} \mathrm{H}$ NMR experiments or $500 \mathrm{mM}$ of TMSP-D 4 for $2 \mathrm{D}^{1} \mathrm{H}_{-}{ }^{13} \mathrm{C}$ HSQC experiments. Samples were then transferred to $5 \mathrm{~mm}$ NMR tubes for data collection.

\section{NMR data collection}

1D and 2D NMR data collection and analysis were performed as described previously. ${ }^{13,14}$ Briefly, all NMR spectra were collected on a Bruker Avance DRX $500 \mathrm{MHz}$ spectrometer equipped with a 5 -mm triple-resonance $\left({ }^{1} \mathrm{H},{ }^{13} \mathrm{C},{ }^{15} \mathrm{~N}\right)$, Z-axis gradient cryoprobe.

Automated data collection utilized a Bruker ATM unit for automatic tuning and matching, a BACS-120 sample changer and Bruker Icon NMR software. The 1D ${ }^{1} \mathrm{H}$ NMR spectra were collected using excitation sculpting for water suppression. ${ }^{15}$ Six replicates were acquired at $298.15 \mathrm{~K}$ with 128 scans, $16 \mathrm{~K}$ data points, 16 dummy scans, a relaxation delay of $1.5 \mathrm{~s}$, a spectral width of $5000 \mathrm{~Hz}$, and a total acquisition time of approximately 7 minutes. $2 \mathrm{D}{ }^{1} \mathrm{H}^{13} \mathrm{C}$ HSQC NMR spectra were collected at $298.15 \mathrm{~K}$ with 128 scans and a relaxation delay of $1.5 \mathrm{~s}$. The spectra were collected with $2 \mathrm{~K}$ data points and a spectrum width of 5,000 $\mathrm{Hz}$ in the direct dimension, and 64 data points and a spectrum width of $17,605.6 \mathrm{~Hz}$ in the indirect dimension, and a total acquisition time of approximately 4 hours. The $2 \mathrm{D}^{1} \mathrm{H}_{-}{ }^{13} \mathrm{C}$ HSQC NMR spectra were processed in NMRPipe and analyzed with NMRViewJ version 9. 16,17

\section{Multivariate Statistical Analysis}

The 1D ${ }^{1} \mathrm{H}$ NMR spectra were processed in our MVAPACK software suite (http:// bionmr.unl.edu/mvapack.php). ${ }^{18}$ For principal component analysis (PCA) and orthogonal projections to latent structures discriminant analysis (OPLS-DA), all spectra were processed with a $1.0 \mathrm{~Hz}$ exponential apodization function prior to being Fourier transformed, automatically phased and normalized using our phase-scatter correction (PSC), ${ }^{19}$ referenced to TMSP-D 4 (0.0 ppm), and scaled using Pareto scaling. For PCA, spectra were binned using an adaptive intelligent binning algorithm that minimizes splitting signals between multiple bins. ${ }^{20}$ Spectral regions containing noise or solvent signals were removed manually. ${ }^{21}$ For OPLS-DA, full-resolution spectra were used to build the model after alignment with the icoshift algorithm ${ }^{22}$ implemented in MVAPACK. OPLS-DA models were calculated using one predictive and one orthogonal component and were cross-validated using a Monte Carlo leave- $n$-out (MCCV) procedure. ${ }^{23}$ The $R^{2}$ (degree of fit) and $\mathrm{Q}^{2}$ (predictive ability) metrics of 0.99236 and 0.97135 for UAMS-1, 0.98905 and 0.96931 for UAMS-1-ackA, and 0.95065 and 0.87454 for the UAMS-1-pta, respectively, indicated highquality OPLS-DA models. Model validation by CV-ANOVA ${ }^{24}$ indicated reliable models with $\mathrm{p}$ values of $3.1 \times 10^{-3}, 2.6 \times 10^{-4}$, and $7.3 \times 10^{-5}$ for the UAMS-1, UAMS-1-ackA and UAMS-1-pta data, respectively. For all strain comparisons, response permutation tests for OPLS-DA model validation also returned $\mathrm{p}$ values of less than 0.01 . The OPLS-DA scores plots and back-scaled loadings were calculated using the entire NMR spectrum (no binning). In this manner, the back-scaled loadings plot resembles a traditional $1 \mathrm{D}{ }^{1} \mathrm{H}$ NMR spectrum, 
where peaks (metabolites) abundant under anaerobic conditions are positive and peaks (metabolites) abundant under aerobic conditions are negative.

\section{Metabolite identification}

The metabolites significantly contributing to class separation in the OPLS-DA scores plot were identified using the Chenomx NMR Suite 8.0. Simply, the experimental 1D ${ }^{1} \mathrm{H}$ NMR spectra were assigned using the Chenomx software and then the back-scaled loadings plot was overlaid with the assigned $1 \mathrm{D}{ }^{1} \mathrm{H}$ NMR spectrum to assign the major features in the back-scaled loadings plot.

Metabolite identification using 2D NMR datasets was performed as described previously. ${ }^{13,14}$ Briefly, the $2 \mathrm{D}{ }^{1} \mathrm{H}^{13} \mathrm{C}$ HSQC NMR spectra were referenced to TMSP-D ${ }_{4}$, and lists of chemical shifts were submitted to the Human Metabolome Database (HMDB), ${ }^{25}$ and the Platform for RIKEN Metabolomics PRIMe, ${ }^{26}$ for metabolite identification. An error tolerance of $0.08 \mathrm{ppm}$ and $0.25 \mathrm{ppm}$, for ${ }^{1} \mathrm{H}$ and ${ }^{13} \mathrm{C}$, respectively, was used to assign the metabolites.

\section{Relative metabolite concentration changes}

$2 \mathrm{D}{ }^{1} \mathrm{H}_{-}{ }^{13} \mathrm{C}$ HSQC NMR spectra were used to measure relative metabolite concentration changes by comparing HSQC peak intensities between spectra collected for the wild-type strain UAMS-1 and the mutant strains UAMS-1-ackA and UAMS-1-pta. The calculation of relative metabolite concentration changes was performed as described previously. ${ }^{13}, 14$ Briefly, a $2 \mathrm{D}{ }^{1} \mathrm{H}^{-13} \mathrm{C}$ HSQC NMR spectrum was collected for each of the three biological replicates obtained for each group (e.g., UAMS-1). The $2 \mathrm{D}^{1} \mathrm{H}_{-}{ }^{13} \mathrm{C}$ HSQC spectra were all referenced to TMSP-D 4 . Each individual $2 \mathrm{D}^{1} \mathrm{H}_{-}{ }^{13} \mathrm{C}$ HSQC NMR spectrum was then normalized to the sum of peak intensities for that specific spectrum. The peak intensities where then scaled, from 1 to 100 , across the entire HSQC dataset, for each individual peak (e.g., ATP HSQC peak: ${ }^{13} \mathrm{C} 98.6 \mathrm{ppm},{ }^{1} \mathrm{H} 6.10 \mathrm{ppm}$ ). An average peak intensity was calculated for each of these HSQC peaks per group. If multiple HSQC peaks were assigned to a single metabolite, then an average of all the HSQC peaks assigned to a metabolite were reported. A paired Student's t-test was utilized to determine the statistical significance of metabolite concentration changes between aerobic and anaerobic growth conditions.

\section{RESULTS}

\section{Anaerobic fermentation partially restores viability of the pta and ackA mutants}

Growth of the pta and ack $A$ mutants under anaerobic (fermentative) conditions provided an important basis for understanding the physiological defects associated with inactivation of the Pta-AckA pathway. Similar to their aerobic growth characteristics, fermentation by both pta and ackA mutants was accompanied by impaired growth rates (Fig 1A, S1), decreased acidification (Fig S1) and a reduction in the levels of excreted acetate relative to the wildtype strain (Fig 1C, S1). Further, an increase in glucose uptake from the culture media was also evident for both the pta and ack $A$ mutants under aerobic and fermentative growth (Fig 1B). Yet surprisingly, both the pta and ack $A$ mutants exhibited a partial restoration of viability (increase in colony forming units ( $\mathrm{cfu}$ ) per unit $\mathrm{OD}_{600}$ ) relative to the wild-type 
strain under fermentative conditions (Fig 1D), despite a decrease in their intracellular ATP levels (Fig 1E). These observations not only suggest a role for fermentative metabolism in increasing survival of the pta and ack $A$ mutants, but also discount perturbations in intracellular ATP as a source of the observed differences in viability of these mutants. Although both aerobic and anaerobic-fermentation resulted in poor growth of the pta and ack $A$ mutants, only the latter fermentative growth conditions led to restoration of cell viability (Fig 1D). This suggests that the relative decrease in growth rate exhibited by the pta and ack $A$ mutants to that of the wild-type strain does not result from increased cell death following inactivation of the Pta-AckA pathway.

\section{Redox homeostasis in the pta and ackA mutants is restored under fermentative conditions}

To further address the differences in viability observed under different oxygen tensions, we utilized 1D ${ }^{1} \mathrm{H}$ NMR to compare the exponential phase metabolomes of the pta and ackA mutants to its isogenic wild-type strain grown under both aerobic and fermentative conditions. Following acquisition of the $1 \mathrm{D}{ }^{1} \mathrm{H}$ NMR spectra, the table of integrals was used for principal component analysis (PCA). Although the PCA showed significant separation between the wildtype strain and corresponding pta and ack $A$ mutants under both aerobic and fermentative conditions, a less pronounced separation was observed following fermentation, suggesting that fermentative metabolism (as opposed to aerobic metabolism) may be less prone to perturbations associated with inactivation of the Pta-AckA pathway (Fig 2A). We reasoned that metabolic compensations in the pta and ack $A$ mutants relative to the wild-type strain may have augmented its survival during fermentation. To identify these metabolic differences, we performed orthogonal partial least squares discriminant analysis (OPLSDA), which relies on class membership, where the variation between classes are represented in the predictive component on the $\mathrm{X}$-axis $(\mathrm{Pp})$ and all other variations are represented in the orthogonal component Y-axis (Po). Multiple metabolites including branched-chain amino acids (valine/isoleucine/leucine), ethanol, lactate, alanine, acetate, betaine, glutamate, aspartate, glycerol, formate and homoserine allowed discrimination of the pta and ack $A$ mutant metabolomes from that of their isogenic wild-type strain under fermentative conditions (Fig 2B and C). However, among these metabolites only lactate, betaine, formate and aspartate appeared to be common to and in excess in both pta and ack $A$ mutants relative to the wild type strain (Fig 2B and C). Although these latter metabolites appear to be unrelated, metabolic pathways involved in their biosynthesis or degradation are potential contributors to cellular redox status during fermentation.

To validate any effects cellular redox status may have on cell viability of the pta and ack $A$ mutants, we next tracked metabolic changes within these mutants by $2 \mathrm{D}{ }^{1} \mathrm{H}-{ }^{13} \mathrm{C}$ HSQC NMR following supplementation of the media with labeled ${ }^{13} \mathrm{C}_{6}$ glucose (see Materials and Methods). Exponentially growing bacteria were collected and used in the $2 \mathrm{D}^{1} \mathrm{H}_{-}{ }^{13} \mathrm{C} \mathrm{HSQC}$ NMR experiments to identify metabolic changes associated with inactivation of the PtaAckA pathway during aerobic and anaerobic growth (Fig S2). Relative changes in the metabolite concentrations were inferred from peak-height differences in the $2 \mathrm{D}^{1} \mathrm{H}_{-}{ }^{13} \mathrm{C}$ HSQC spectra (Table S1). Consistent with the results of PCA and OPLS-DA, the $2 \mathrm{D}^{1} \mathrm{H}_{-}{ }^{13} \mathrm{C}$ HSQC NMR experiments revealed broad similarities in the metabolic changes between the wild-type, pta and ackA mutant strains during fermentation (Fig 3A). More specifically, a 
decrease in the relative concentrations of the TCA cycle intermediates as well as metabolites associated with its activity were observed for all bacterial cultures under anoxic growth conditions (Fig 3A and B). Moreover, in contrast to aerobic growth, where inactivation of the Pta-AckA pathway caused redirection of carbon flux into the TCA cycle as indicated by an increase in intracellular levels of citrate, succinate, a-ketoglutarate, and glutamate, ${ }^{10}$ no differences in the relative concentrations of these metabolites between the wild-type, pta and ack $A$ mutants were detected during fermentation (Fig 3A and B). Furthermore, whereas a marked increase in the intracellular pools of $\mathrm{NAD}^{+}$and $\mathrm{NADH}$ was observed following inactivation of the Pta-AckA pathway under aerobic growth, fermentation by the pta and ack $A$ mutants resulted in a significant decrease in the intracellular concentrations for both of these species (Fig 3A) consistent with inhibition of the TCA cycle activity under anaerobiosis. Additionally, the relative intracellular concentrations of $\mathrm{NAD}^{+}$and $\mathrm{NADH}$ were similar between the mutants and the wild-type strain suggestive of a lower energy status for all strains under anoxic conditions (Fig 3A).

In addition to the loss of the TCA cycle activity, previous studies have demonstrated that the redirection of carbon flux into D- and L-lactate generation plays an important role in the maintenance of redox balance in $S$. aureus during anaerobic growth as these pathways are required to replenish the supply of $\mathrm{NAD}^{+}$for glycolysis to proceed. ${ }^{27-32}$ Consistent with these studies, we detected higher levels of intracellular lactate in all bacteria by $2 \mathrm{D}^{1} \mathrm{H}^{-13} \mathrm{C}$ HSQC NMR during anaerobiosis (Fig 3A and B). However, when compared to the wild-type strain, we observed decreased concentrations of intracellular lactate in the pta and ack $A$ mutants under fermentative growth (Fig 3A). Although this may appear counterintuitive for both the pta and ack $A$ mutants that require increased turnover of NADH to NAD ${ }^{+}$to maintain redox homeostasis, this phenotype did not result from a lack of lactate production. Rather, we observed an increased excretion of both D- and L-lactate by the pta and ack $A$ mutants relative to the wild-type strain, suggesting that the decreased intracellular levels of lactate reflected increased turnover and export of carbon through the lactate dehydrogenase pathway in these mutants (Fig 1F). Collectively, these results indicate that achieving redox homeostasis may be crucial to increasing viability of the pta and ack $A$ mutants.

\section{Overexpression of the L-lactate dehydrogenase (Ldh1) increases viability of the pta mutant during aerobic growth}

Given that the increased activation of fermentative pathways (D- and L-lactate) during anaerobiosis potentially allows $S$. aureus pta and ack $A$ mutants to maintain redox homeostasis and viability, we argued that a similar correction of the redox status following inactivation of the Pta-AckA pathway under aerobic growth should prevent cell death. To test this hypothesis, we introduced a plasmid overexpressing L-lactate dehydrogenase (pMRS110) into the wild-type strain and the pta mutant and monitored cell viability and Llactate production during aerobic growth. As expected, overexpression of the $1 d h 1$ gene caused a significant increase in the excretion of L-lactate for both the wild-type strain and pta mutant (Fig. 4A). However, excreted L-lactate concentrations in the media were higher for the pta mutant than the wild-type strain (Fig. 4A), presumably due to increased glycolytic flux associated with disruption of the Pta-AckA pathway. More importantly, while pMRS110 mediated overexpression of the $1 d h 1$ increased viability of the pta mutant relative 
to the control strain (pta mutant bearing vector control, pBK123), no differences in the viable cell counts were observed for the wild-type strain carrying either of these plasmids (Fig. 4B). Overall, these results demonstrate that restoration of redox homeostasis can significantly attenuate the negative impact on cell viability associated with inactivation of the Pta-AckA pathway under aerobic growth conditions.

\section{DISCUSSION}

During aerobic growth in media containing glucose, when carbon flux towards the TCA cycle is limited due to carbon catabolite repression, ${ }^{33-37}$ S. aureus primarily generates acetate by means of the Pta-AckA pathway, a phenomenon known as "acetate overflow". ${ }^{10,30,32,38,39}$ We have previously shown that under these conditions the PtaAckA pathway plays an indispensable role in maintenance of $S$. aureus fitness, as its inactivation results in growth defects and cell death. Although the precise reasons for the observed defects in growth and viability of pta and ack $A$ mutants are not clearly understood, a role for ATP production (a byproduct of the Pta-AckA pathway) was ruled out earlier. ${ }^{10}$ Interestingly, similar observations were also noted in other bacteria, i.e. it was shown that disruption of the pta gene in Escherichia colit ${ }^{40}$ and inactivation of the ack $A$ gene in Streptococcus mutans ${ }^{41}$ did not cause a substantial loss in the amounts of generated ATP.

How do the $S$. aureus pta and ack $A$ mutants compensate for the loss of ATP following inactivation of the Pta-AckA pathway? The results of the current study suggest that disruption of this pathway under aerobic conditions increases glycolytic flux and redirects carbon towards the TCA cycle. This was reflected by a higher glucose consumption rate and by increased intracellular concentrations of glycolytic and TCA cycle intermediates. Additionally, transcriptional upregulation of key glycolytic and TCA cycle genes i.e., $p f k A$ and $c i t Z$ was also observed in the pta and ack $A$ mutants. ${ }^{10} \mathrm{~A}$ similar increase in the expression of both TCA cycle and central glycolytic enzymes in the Pta-AckA pathway mutants was reported for E. coli. ${ }^{42,}{ }^{43} \mathrm{An}$ increase in carbon flux through both glycolysis and the TCA cycle could compensate for ATP loss following inactivation of the Pta-AckA pathway, since sufficient reducing equivalents generated through these pathways will be converted to ATP by means of oxidative phosphorylation. ${ }^{44}$ In support of this argument, both respiration rates and intracellular $\mathrm{NAD}^{+}$and $\mathrm{NADH}$ concentrations were observed to be significantly higher in the pta and ack $A$ mutants. ${ }^{10}$

Both $\mathrm{NAD}^{+}$and NADH are the primary determinants of redox balance in living cells and their intracellular concentrations are crucial for the sustained function of a variety of metabolic pathways. Hence, any compensatory increase in flux through glycolysis and TCA cycle raises the potential risk of a redox imbalance in cells. Indeed, we speculated that the altered redox environment associated with the pta and ack $A$ mutations might contribute to the observed growth and viability defects in these mutants. Evidence supporting this notion was observed when reduced viability, a characteristic of both pta and ack $A$ mutant populations, was partially restored to wild-type levels following growth under fermentative conditions. Fermentation by pta and ack $A$ mutants not only prevented redirection of carbon towards the TCA cycle (as this pathway is inactive under anoxic conditions), but apparently also resulted in a balanced intracellular turnover of NADH to NAD ${ }^{+}$. NMR metabolomics 
analysis and metabolite excretion profiles of anaerobically grown pta and ack $A$ mutants identified multiple possibilities that could aid in the turnover of intracellular pools of NADH and $\mathrm{NAD}^{+}$in these mutants. Specifically, whereas biosynthesis of betaine from betaine aldehyde, and aspartate from glutamate would result in the generation of NADH, the conversion of pyruvate to lactate increases turnover of NADH to NAD ${ }^{+}$. Similarly, excess levels of formate in the cells may be indicative of decreased formate dehydrogenase activity, an adaptive strategy that could potentially limit excess NADH production from NAD ${ }^{+}$. These observations broadly correlate activity of reactions that augment cellular redox homeostasis to increased viability of the pta and ack $A$ mutants under fermentative conditions.

Confirmatory evidence linking the altered intracellular redox status and decreased viability following inactivation of the Pta-AckA pathway could be established only after overexpressing L-lactate dehydrogenase in the $S$. aureus pta mutant. $S$. aureus possesses two L-lactate dehydrogenases (Ldh1/2) and two D-lactate dehydrogenases (SACOL2535 and SACOL2574). ${ }^{30,45,46}$ However, these enzymes are generally not active under aerobic conditions. ${ }^{31}$ Overexpression of $1 d h 1$ in the pta mutant using an inducible promoter partially restored its viability to wild-type levels under aerobic conditions. Interestingly, this approach did not restore the decreased growth rate of the pta mutant (data not shown) suggesting redoxindependent regulation of growth following inactivation of the Pta-AckA pathway. An alternate possibility is that restoration of redox homeostasis in the pta mutant by overexpression of $1 d h 1$ may decrease the intracellular pools of ATP leading to defects in growth rate.

In conclusion, it has often been argued that in glucose-rich environments, carbon flux through the Pta-AckA pathway is a rapid means by which $S$. aureus generates ATP to support fast growth under aerobic conditions. Although this may be true in principle, our data clearly suggests that the Pta-AckA pathway may have an alternate role in maintaining redox homeostasis by diverting carbon away from the TCA cycle. The inability to achieve this balance results in growth defects and cell death due to redox toxicity. Given that multiple bacterial species not only possess the Pta-AckA pathway, but also exhibit similar physiological phenotypes following its inactivation, we argue that the ability to maintain redox homeostasis by directing carbon through the Pta-AckA pathway may be a universal phenomenon.

\section{Supplementary Material}

Refer to Web version on PubMed Central for supplementary material.

\section{Acknowledgments}

This manuscript was supported by National Institute of Health grants P30 GM103335 (R.P.), P01-AI083211 (K.W.B.), R01-A1038901 (K.W.B.), and from the American Heart Association grant 0860033Z (R.P.). Parts of this research were performed in facilities renovated with support from the National Institutes of Health (RR015468-01). 


\section{References}

1. Lowy FD. Staphylococcus aureus infections. N Engl J Med. 1998; 339(8):520-32. [PubMed: 9709046]

2. Chambers HF, Deleo FR. Waves of resistance: Staphylococcus aureus in the antibiotic era. Nat Rev Microbiol. 2009; 7(9):629-41. [PubMed: 19680247]

3. Wertheim HF, Vos MC, Ott A, van Belkum A, Voss A, Kluytmans JA, van Keulen PH, Vandenbroucke-Grauls CM, Meester MH, Verbrugh HA. Risk and outcome of nosocomial Staphylococcus aureus bacteraemia in nasal carriers versus noncarriers. Lancet. 2004; 364(9435): 703-5. [PubMed: 15325835]

4. Novick RP. Autoinduction and signal transduction in the regulation of staphylococcal virulence. Mol Microbiol. 2003; 48(6):1429-49. [PubMed: 12791129]

5. Seidl K, Stucki M, Ruegg M, Goerke C, Wolz C, Harris L, Berger-Bachi B, Bischoff M. Staphylococcus aureus CcpA affects virulence determinant production and antibiotic resistance. Antimicrob Agents Chemother. 2006; 50(4):1183-94. [PubMed: 16569828]

6. Majerczyk CD, Sadykov MR, Luong TT, Lee C, Somerville GA, Sonenshein AL. Staphylococcus aureus CodY negatively regulates virulence gene expression. J Bacteriol. 2008; 190(7):2257-65. [PubMed: 18156263]

7. Chaffin DO, Taylor D, Skerrett SJ, Rubens CE. Changes in the Staphylococcus aureus transcriptome during early adaptation to the lung. PLoS One. 2012; 7(8):e41329. [PubMed: 22876285]

8. Cheung AL, Bayer AS, Zhang G, Gresham H, Xiong YQ. Regulation of virulence determinants in vitro and in vivo in Staphylococcus aureus. FEMS Immunol Med Microbiol. 2004; 40(1):1-9. [PubMed: 14734180]

9. Richardson AR, Somerville GA, Sonenshein AL. Regulating the Intersection of Metabolism and Pathogenesis in Gram-positive Bacteria. Microbiol Spectr. 2015; 3(3)

10. Sadykov MR, Thomas VC, Marshall DD, Wenstrom CJ, Moormeier DE, Widhelm TJ, Nuxoll AS, Powers R, Bayles KW. Inactivation of the Pta-AckA pathway causes cell death in Staphylococcus aureus. J Bacteriol. 2013; 195(13):3035-44. [PubMed: 23625849]

11. Sharma-Kuinkel BK, Mann EE, Ahn JS, Kuechenmeister LJ, Dunman PM, Bayles KW. The Staphylococcus aureus LytSR two-component regulatory system affects biofilm formation. J Bacteriol. 2009; 191(15):4767-75. [PubMed: 19502411]

12. Meyer H, Liebeke M, Lalk M. A protocol for the investigation of the intracellular Staphylococcus aureus metabolome. Anal Biochem. 2010; 401(2):250-259. [PubMed: 20211591]

13. Halouska S, Zhang B, Gaupp R, Lei S, Snell E, Fenton RJ, Barletta RG, Somerville GA, Powers R. Revisiting Protocols for the NMR Analysis of Bacterial Metabolomes. J Integr OMICS. 2013; 3(2):120-137. [PubMed: 26078915]

14. Zhang B, Halouska S, Schiaffo CE, Sadykov MR, Somerville GA, Powers R. NMR analysis of a stress response metabolic signaling network. J Proteome Res. 2011; 10(8):3743-54. [PubMed: 21692534]

15. Simpson AJ, Brown SA. Purge NMR: effective and easy solvent suppression. J Magn Reson. 2005; 175(2):340-6. [PubMed: 15964227]

16. Delaglio F, Grzesiek S, Vuister GW, Zhu G, Pfeifer J, Bax A. NMRPipe: a multidimensional spectral processing system based on UNIX pipes. J Biomol NMR. 1995; 6(3):277-93. [PubMed: 8520220]

17. Johnson BA. Using NMRView to visualize and analyze the NMR spectra of macromolecules. Methods Mol Biol. 2004; 278:313-52. [PubMed: 15318002]

18. Worley B, Powers R. MVAPACK: a complete data handling package for NMR metabolomics. ACS Chem Biol. 2014; 9(5):1138-44. [PubMed: 24576144]

19. Worley B, Powers R. Simultaneous Phase and Scatter Correction for NMR Datasets. Chemometr Intell Lab Syst. 2014; 131:1-6. [PubMed: 24489421]

20. De Meyer T, Sinnaeve D, Van Gasse B, Tsiporkova E, Rietzschel ER, De Buyzere ML, Gillebert TC, Bekaert S, Martins JC, Van Criekinge W. NMR-based characterization of metabolic alterations in hypertension using an adaptive, intelligent binning algorithm. Anal Chem. 2008; 80(10):3783-90. [PubMed: 18419139] 
21. Halouska S, Powers R. Negative impact of noise on the principal component analysis of NMR data. J Magn Reson. 2006; 178(1):88-95. [PubMed: 16198132]

22. Savorani F, Tomasi G, Engelsen SB. Icoshift: A versatile tool for the rapid alignment of 1D NMR spectra. J Magn Reson. 2010; 202(2):190-202. [PubMed: 20004603]

23. Xu Q-S, Liang Y-Z, Du Y-P. Monte Carlo cross-validation for selecting a model and estimating the prediction error in multivariate calibration. J Chemometrics. 2004; 18:112-120.

24. Eriksson L, Trygg J, Wold S. CV-ANOVA for significance testing of PLS and OPLS® models. J Chemometrics. 2008; 22:594-600.

25. Wishart DS, Jewison T, Guo AC, Wilson M, Knox C, Liu Y, Djoumbou Y, Mandal R, Aziat F, Dong E, Bouatra S, Sinelnikov I, Arndt D, Xia J, Liu P, Yallou F, Bjorndahl T, Perez-Pineiro R, Eisner R, Allen F, Neveu V, Greiner R, Scalbert A. HMDB 3.0-The Human Metabolome Database in 2013. Nucleic Acids Res. 2013; 41:D801-7. (Database issue). [PubMed: 23161693]

26. Akiyama K, Chikayama E, Yuasa H, Shimada Y, Tohge T, Shinozaki K, Hirai MY, Sakurai T, Kikuchi J, Saito K. PRIMe: a Web site that assembles tools for metabolomics and transcriptomics. In Silico Biol. 2008; 8(3-4):339-45. [PubMed: 19032166]

27. Garvie EI. Bacterial lactate dehydrogenases. Microbiol Rev. 1980; 44(1):106-39. [PubMed: 6997721]

28. Ojima Y, Suryadarma P, Tsuchida K, Taya M. Accumulation of pyruvate by changing the redox status in Escherichia coli. Biotechnol Lett. 2012; 34(5):889-93. [PubMed: 22215378]

29. Sun JL, Zhang SK, Chen JY, Han BZ. Metabolic profiling of Staphylococcus aureus cultivated under aerobic and anaerobic conditions with (1)H NMR-based nontargeted analysis. Can J Microbiol. 2012; 58(6):709-18. [PubMed: 22571732]

30. Ferreira MT, Manso AS, Gaspar P, Pinho MG, Neves AR. Effect of oxygen on glucose metabolism: utilization of lactate in Staphylococcus aureus as revealed by in vivo NMR studies. PLoS One. 2013; 8(3):e58277. [PubMed: 23472168]

31. Fuchs S, Pane-Farre J, Kohler C, Hecker M, Engelmann S. Anaerobic gene expression in Staphylococcus aureus. J Bacteriol. 2007; 189(11):4275-89. [PubMed: 17384184]

32. Ledala N, Zhang B, Seravalli J, Powers R, Somerville GA. Influence of iron and aeration on Staphylococcus aureus growth, metabolism, and transcription. J Bacteriol. 2014; 196(12):2178-89. [PubMed: 24706736]

33. Crabtree HG. Observations on the carbohydrate metabolism of tumours. Biochem J. 1929; 23(3): 536-45. [PubMed: 16744238]

34. Sonenshein AL. Control of key metabolic intersections in Bacillus subtilis. Nat Rev Microbiol. 2007; 5(12):917-27. [PubMed: 17982469]

35. Seidl K, Muller S, Francois P, Kriebitzsch C, Schrenzel J, Engelmann S, Bischoff M, Berger-Bachi B. Effect of a glucose impulse on the CcpA regulon in Staphylococcus aureus. BMC Microbiol. 2009; 9:95. [PubMed: 19450265]

36. Sadykov MR, Hartmann T, Mattes TA, Hiatt M, Jann NJ, Zhu Y, Ledala N, Landmann R, Herrmann M, Rohde H, Bischoff M, Somerville GA. CcpA coordinates central metabolism and biofilm formation in Staphylococcus epidermidis. Microbiology. 2011; 157(Pt 12):3458-68. [PubMed: 21964732]

37. Hanson RS, Cox DP. Effect of different nutritional conditions on the synthesis of tricarboxylic acid cycle enzymes. J Bacteriol. 1967; 93(6):1777-87. [PubMed: 4960893]

38. Majewski RA, Domach MM. Simple constrained-optimization view of acetate overflow in E coli. Biotechnol Bioeng. 1990; 35(7):732-8. [PubMed: 18592570]

39. Somerville GA, Proctor RA. At the crossroads of bacterial metabolism and virulence factor synthesis in Staphylococci. Microbiol Mol Biol Rev. 2009; 73(2):233-48. [PubMed: 19487727]

40. Chang DE, Shin S, Rhee JS, Pan JG. Acetate metabolism in a pta mutant of Escherichia coli W3110: importance of maintaining acetyl coenzyme A flux for growth and survival. J Bacteriol. 1999; 181(21):6656-63. [PubMed: 10542166]

41. Kim JN, Ahn SJ, Burne RA. Genetics and Physiology of Acetate Metabolism by the Pta-Ack Pathway of Streptococcus mutans. Appl Environ Microbiol. 2015; 81(15):5015-25. [PubMed: 25979891] 
42. Wolfe AJ, Chang DE, Walker JD, Seitz-Partridge JE, Vidaurri MD, Lange CF, Pruss BM, Henk MC, Larkin JC, Conway T. Evidence that acetyl phosphate functions as a global signal during biofilm development. Mol Microbiol. 2003; 48(4):977-88. [PubMed: 12753190]

43. Wolfe AJ. The acetate switch. Microbiol Mol Biol Rev. 2005; 69(1):12-50. [PubMed: 15755952]

44. Mitchell P, Moyle J. Chemiosmotic hypothesis of oxidative phosphorylation. Nature. 1967; 213(5072):137-9. [PubMed: 4291593]

45. Stockland AE, San Clemente CL. Multiple forms of lactate dehydrogenase in Staphylococcus aureus. J Bacteriol. 1969; 100(1):347-53. [PubMed: 4310081]

46. Boyle-Vavra S, de Jonge BL, Ebert CC, Daum RS. Cloning of the Staphylococcus aureus ddh gene encoding NAD+-dependent D-lactate dehydrogenase and insertional inactivation in a glycopeptide-resistant isolate. J Bacteriol. 1997; 179(21):6756-63. [PubMed: 9352927] 
A

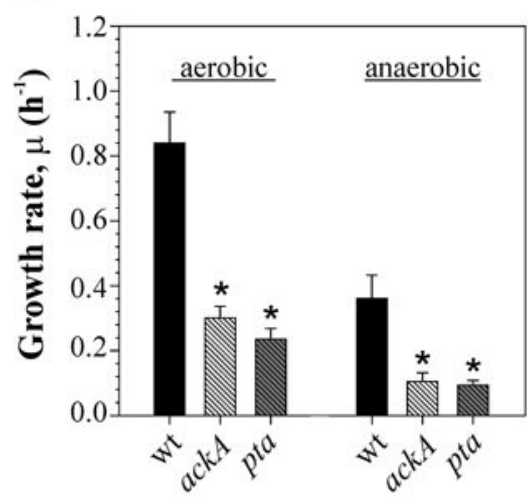

D

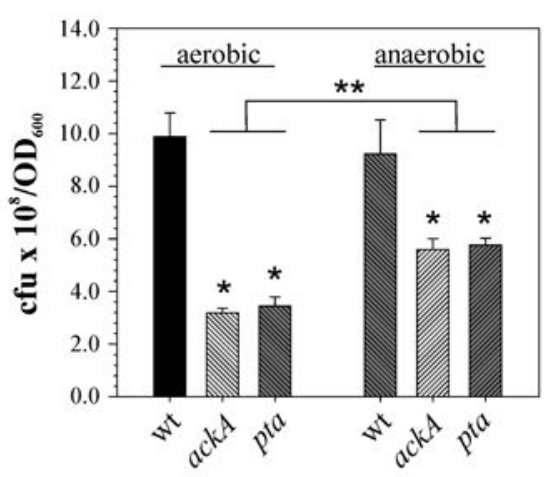

B

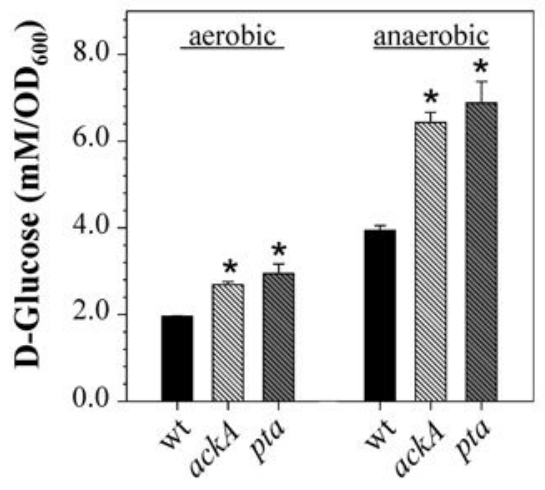

E

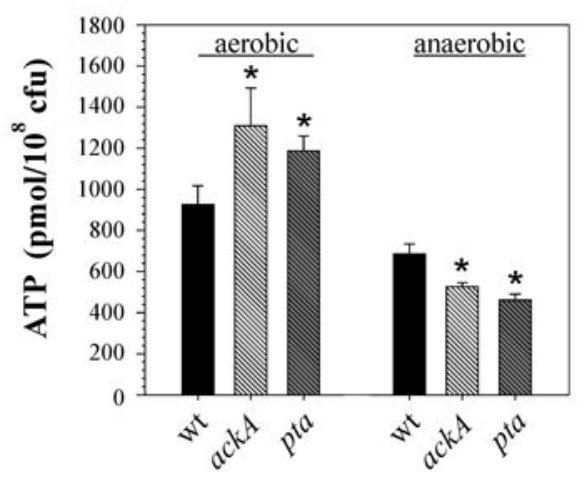

C

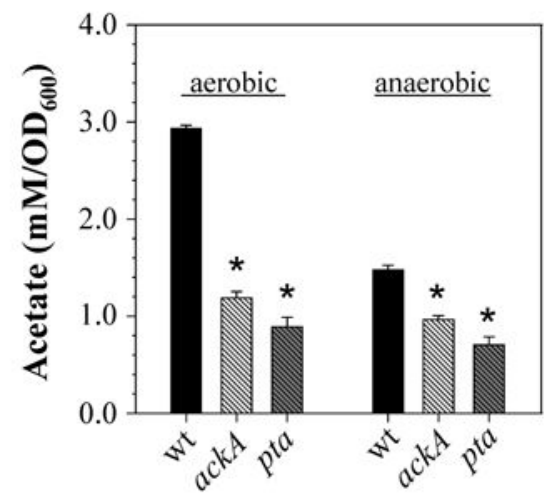

$\mathbf{F}$

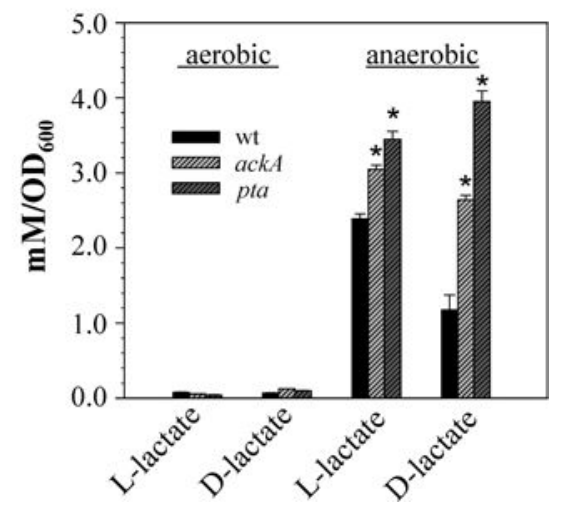

Figure 1. Inactivation of the Pta-AckA pathway affects growth characteristics and alters viability and energy status of $S$. aureus during aerobic and anaerobic growth

(A) Growth rate $\mu$ (hour ${ }^{-1}$ ) of the wild type strain UAMS-1, and mutant strains UAMS-1ack $A$, and UAMS-1-pta grown aerobically or anaerobically in TSB containing $0.25 \%$

glucose determined after 3 hours of growth. (B) The concentrations of glucose consumed from the culture medium per $\mathrm{OD}_{600}$ determined for strains UAMS-1, UAMS-1-ackA and UAMS-1-pta after 3 hours of growth. (C) The concentrations of accumulated acetic acid in the culture medium per $\mathrm{OD}_{600}$ determined for strains UAMS-1, UAMS-1-ackA and

UAMS-1-pta after 3 hours of growth. (D) Number of viable cells per OD $_{600}$ unit determined for strains UAMS-1, UAMS-1-ackA, and UAMS-1-pta after $3 \mathrm{~h}$ of growth. (E) Intracellular ATP concentrations determined for strains UAMS-1, UAMS-1-ackA, and UAMS-1-pta after $3 \mathrm{~h}$ of growth. (F) The concentrations of accumulated D- and L-lactic acids in the culture medium per $\mathrm{OD}_{600}$ determined for strains UAMS-1, UAMS-1-ackA and UAMS-1-pta after 3 hours of growth. The results are presented as the means plus standard errors of the mean of duplicate determinations for at least three independent experiments. Statistical significance between the wild-type strain and pta and ack $A$ mutants (*) and pta and ack $A$ mutants grown aerobically and anaerobically (**) was determined by Student's $t$ test $(P \leq 0.001)$. 
A
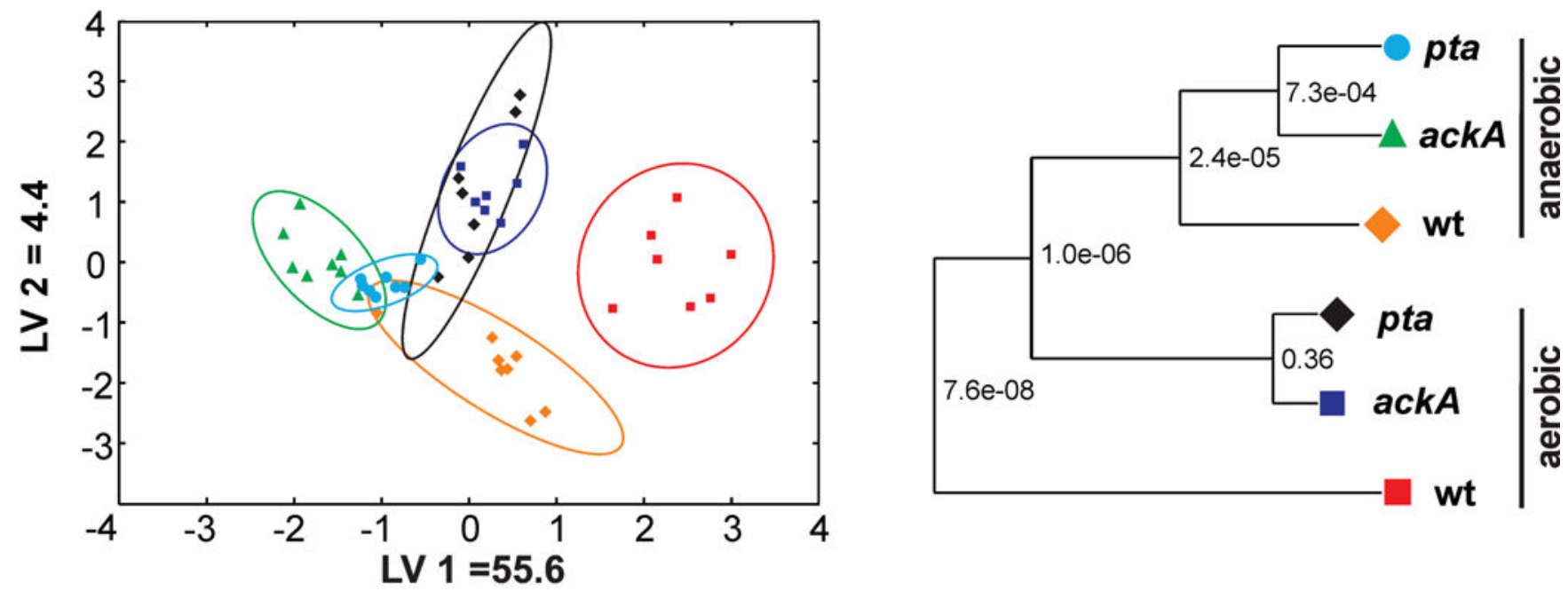

B
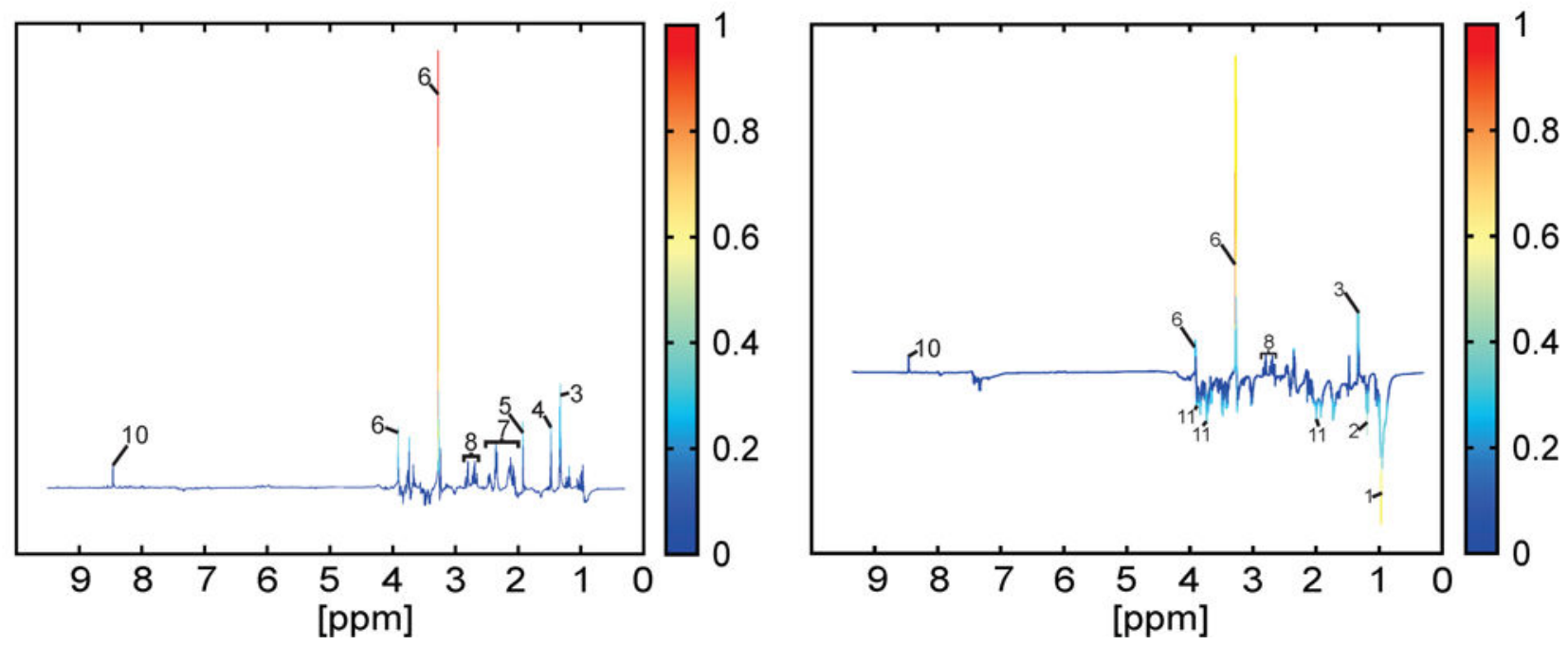

Figure 2. Disruption of the Pta-AckA pathway has relatively smaller impact on S. aureus metabolome during anaerobiosis

(A): 2D LDA scores plots comparing the metabolic fingerprints of aerobically grown wild type (red), UAMS-1-ackA (purple), and UAMS-1-pta (black) vs. anaerobically grown wild type (yellow), UAMS-1-ackA (green), and UAMS-1-pta (cyan). Dendrogram was generated from PCA scores using a Mahalanobis distance matrix with $p$ values for the null hypothesis reported at each node. (B and $\mathbf{C}$ ) OPLS-DA back-scaled loadings plot comparing anaerobically grown UAMS-1-pta (B) and UAMS-1-ackA (D) vs. UAMS-1 wild-type strain. Peak intensities reflect the contribution of that peak to class separation, an up orientation represents a metabolite increased under anaerobic growth, and conversely a down orientation represents a metabolite increased under aerobic growth. The coloring of the backscaled loadings plot is based on the scale factor applied during variable scaling prior to model training. Metabolite labels correspond to: (1) branched chain amino acids (valine/isoleucine/ 
leucine), (2) ethanol, (3) lactate, (4) alanine, (5) acetate, (6) betaine, (7) glutamate, (8) aspartate, (9) glycerol, (10) formate, and (11) homoserine. 


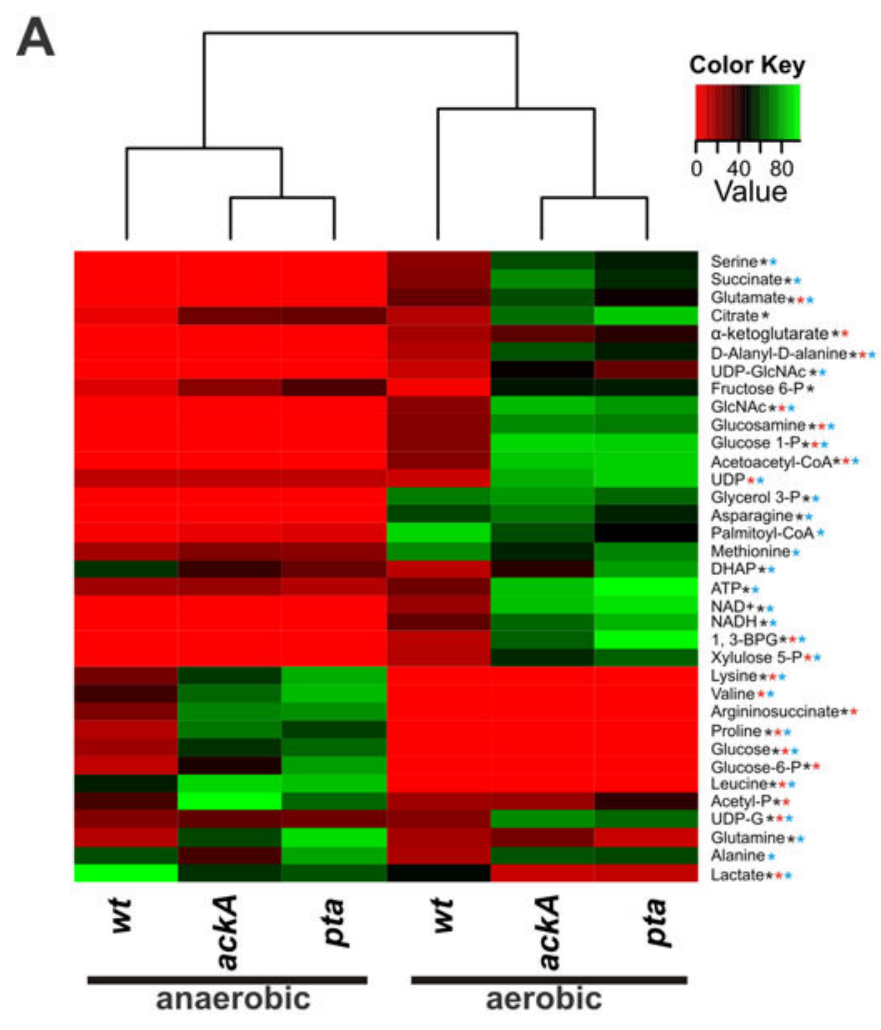

B

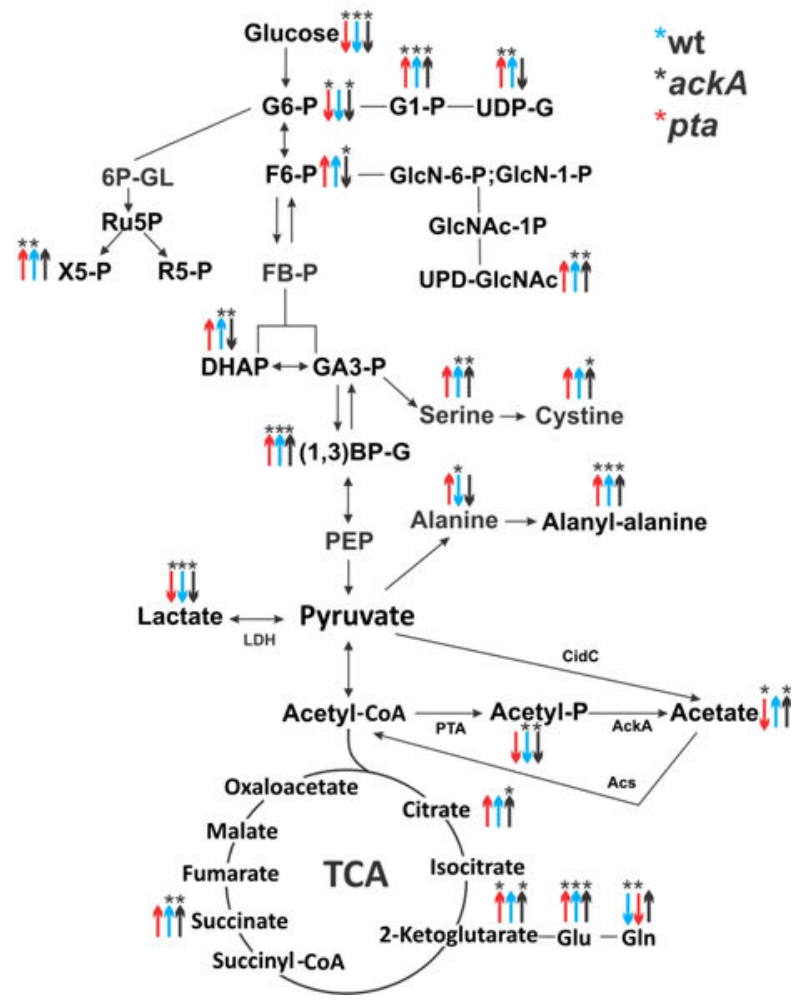

Figure 3. Oxygen availability alters catabolic fate of glucose in the pta and ackA mutants (A) The heat map generated from normalized peak intensities in $2 \mathrm{D}^{1} \mathrm{H}-{ }^{13} \mathrm{C}$ HSQC NMR spectra with the dendrogram representing a hierarchal clustering that compares UAMS-1, UAMS-1-ack $A$, and UAMS-1-pta $S$ aureus grown aerobically or anaerobically. The color scale ranges from 0 (red) (less intense) to 1 (green) (intense) and strain colored asterisks denotes statistical significance at the $95 \%$ confidence comparing aerobic vs. anaerobic conditions. A metabolite that was not observed under either aerobic or anaerobic growth conditions was assigned a value of zero. Please see Table S1 for a complete listing of the normalized peak intensities. (B) Metabolic network summarizes the metabolic perturbations observed comparing UAMS-1, UAMS-1-ackA, and UAMS-1-pta under aerobic vs. anaerobic conditions. Up arrows indicate a relative increase in metabolite concentration, and a down arrow indicates a relative decrease in the metabolite concentration for aerobic compared to anaerobic conditions. The asterisk above each arrow denotes statistical significance at the $95 \%$ confidence level $(\mathrm{p}<0.05)$. Abbreviations are as follows acetylphosphate (Acetyl-P); (1,3)BP-G, 1,3-bisphosphoglycerate; F6-P, fructose 6-phosphate; Glu, glutamate, Gln, glutamine, FB-P, fructose 1,6-bisphosphate; DHAP, dihydroxyacetone phosphate; G6-P, glucose 6-phosphate; G1-P, glucose 1-phosphate; GlcN-6-P, glucosamine6-phosphate; GlcN-1-P, glucosamine-1-phosphate; GA3-P, glyceraldehyde 3-phosphate; GlcNAc-1-P, N-acetyl-glucosamine-1-phosphate; GlcNAc-6-P, N-acetyl-glucosamine-6phosphate; 3P-G, 3-phosphoglycerate; 2P-G, 2-phosphoglycerate; 6P-GL, 6phosphonoglucono-lactone; PEP, phosphoenolpyruvate; Ru5P, ribulose 5-phosphate; UDP$\mathrm{G}$, uridine diphosphate glucose; UDP-GlcNAc, uridine diphosphate N- 
acetylglucosamine; R5P, ribose-5-phosphate; Ru5P, ribulose 5-phosphate; X5-P, xylulose 5phosphate. 


\section{A}

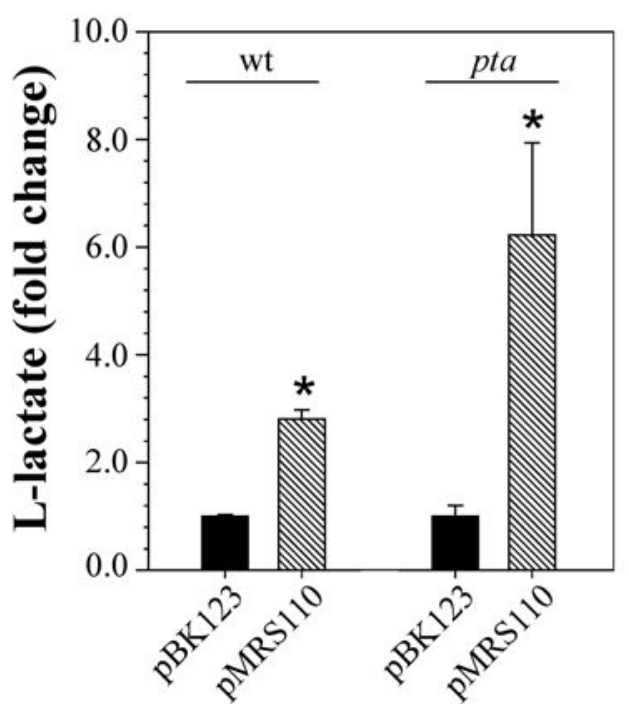

B

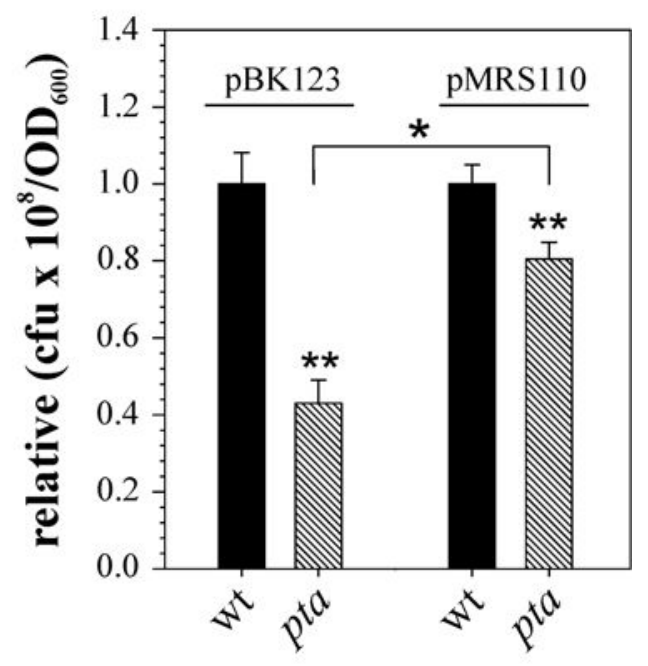

Figure 4. Overexpression of the $l d h 1$ gene attenuates negative impact on viability of the pta mutant during aerobic growth

(A) Relative concentrations of accumulated L-lactic acid in the culture medium per $\mathrm{OD}_{600}$ determined for strains UAMS-1, UAMS-1-ackA and UAMS-1-pta containing vector plasmid (pBK123) or ldh1 expressing plasmid (pMRS110) after 3 hours of growth. (B) Relative number of viable cells per $\mathrm{OD}_{600}$ unit determined for strains UAMS-1, UAMS-1-ackA, and UAMS-1-pta containing vector plasmid (pBK123) or ldh1 expressing plasmid (pMRS110) after $3 \mathrm{~h}$ of growth. The results are presented as the means plus standard errors of the mean of duplicate determinations for at least three independent experiments. Statistical significance between strains containing vector plasmid (pBK123) and ldh1 expressing plasmid (pMRS110) (A and B) and between the wild-type strain and pta and ackA mutants (B) was determined by Student's $t$ test $(* * *, P \leq 0.001)$. 